\title{
Channel Estimation in MIMO-OFDM System
}

\author{
S. R. Aryal and H. Dhungana \\ Institute of Engineering, Tribhuvan University, Pulchowk Campus, Lalitpur \\ e-mail:sapan.614@gmail.com
}

\begin{abstract}
There are no limit of human desire, so day by day we need much higher data speed to facilitate our need but every physical resource like frequency band, transmit signal strength are finite. Within the given limited resource, higher data speed is accomplished by new proficiency called Multiple Input Multiple Output (MIMO), Orthogonal Frequency Division Multiplexing (OFDM) system. MIMO-OFDM fulfills the high data rate requirement through spatial multiplexing gain and improved link reliability due to antenna diversity gain. With this technique, both interference reduction and maximum diversity gain are achieved by increasing number of antennae on either side. Received signal in MIMO-OFDM system is usually distorted by multipath fading. In order to recover the transmitted signal correctly, channel effect must be estimated and repaired at receiver. In this paper the performance evaluating parameter mean square error and symbol error rate of least square error, minimum mean square error and DFT based channel estimation methods are estimated and appropriate solution is recommended. Furthermore, comparison among their characteristics is simulated in MATLAB and useful conclusion is delineated.
\end{abstract}

Key words: mean square error, symbol error rate, least square error, minimum mean square error, Bit error rate

\section{Introduction}

During the past decades, wireless communication has benefitted from substantial advances and it is considered as the key enabling technique of innovative future consumer products. For the sake of satisfying the requirements of various applications, significant technological achievements are required to ensure that wireless devices have appropriate architectures suitable for supporting a wide range of services delivered to the users. In the foreseeable future, the large-scale deployment of wireless devices and the requirements of high bandwidth applications are expected to lead to tremendous new challenges in terms of the efficient exploitation of the achievable spectral resources. Among the existing air-interface techniques, OFDM has shown a number of advantages and has attracted substantial interest. OFDM converts a frequency-selective channel into a parallel collection of frequency flat sub channels. The subcarriers have the minimum frequency separation required to maintain orthogonality of their corresponding time domain waveforms, yet the signal spectra corresponding to the different subcarriers overlap in frequency. Hence, the available bandwidth is very efficiently used. If knowledge of the channel is available at the transmitter, then the OFDM transmitter can adapt its signaling strategy to match the channel. Due to the fact that OFDM uses a large collection of narrowly spaced sub channels, these adaptive strategies can approach the ideal water pouring capacity of a frequency-selective channel. In practice this is achieved by using adaptive bit loading techniques, where different sized signal constellations are transmitted on the subcarriers (Rony Kowalski 2007).

With a drastic increase in number of users feeling comfortable while accessing wireless services in last few years, there is a fair demand for higher system throughputs to accommodate more users with higher data rates. MIMO based OFDM has the potential to achieve high system capacity and transmit-receive diversity for reliable communication links of any wireless system, hence it is considered as the future of wireless communication systems. MIMO OFDM 
systems provide higher data rates, support a large number of users with flexibility in quality of service (QoS) and provide high quality transmission in comparison to other schemes. But in order to fulfill these requirements some constraints have to be very well addressed such as limited availability of frequency spectrum, availability of total transmit power and nature of wireless channels.

The bit error rate, mean square error and symbol error rate are performance evaluating parameters in channel estimation. On the basis of the required accuracy of the estimate and optimal training sequences of minimum length, channel estimators are chosen.This paper begins from the introduction of current wireless communication pattern, followed by wireless channel model. Next section describes the channel estimation techniques followed by simulation of least square channel estimation and minimum mean square error channel estimation. Finally the paperwrappes up by giving some comparisons result, future works for further research and recommendations.

\section{Methodology}

The performance of any communication system is primarily decided by the communication channel. Wireless channel in a communication system are categories into four types called propagation channel, radio channel, modulation channel and digital channel; according to the different instances in the transmission and reception process of signal (Aguiar\& Gross 2003). There are different types of channels among them wireless channel has unreliable behavior; the state of the channel may change within a very short time span. This stochastic and rapid behavior of radio channels turns wireless data communication into a difficult task. Path loss, shadowing and fading are three main factors to influence the received signal on radio channel. Path loss is a deterministic effect

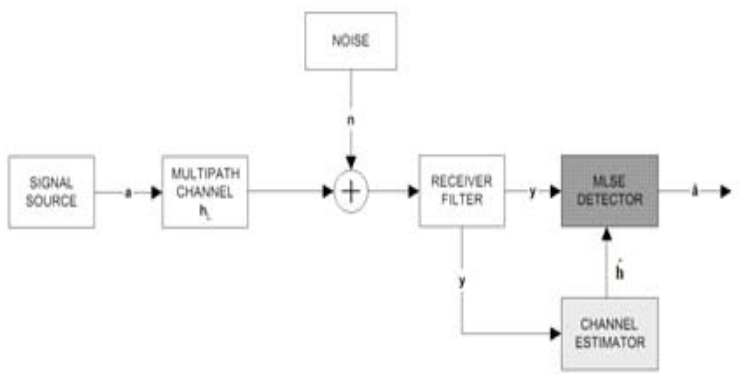

Fig. 1. Block diagram of channel estimation and cause weakening of signal strength depending only on the distance between the transmitter and the receiver. Shadowing fluctuate the received signal strength at points with the same distance to the transmitter. It varies on the same time scale as the path loss does and is not deterministic in nature. Interference due to scattered signals arriving at an antenna is called fading. Fading cause significant attenuation changes in received signal strength within smaller time scales such as milliseconds or even microseconds. It is mostly present in multipath propagation environment, and random in nature. It is responsible for the most rapid and violent changes of the signal strength itself as well as its phase. These signal variations are experienced on a small time scale, mostly a fraction of a second or shorter, depending on the velocity of the receiver. The basis of origination fading in wireless communication is by the reception of multiple copies of the transmitted signal, each having followed a different path. The fading may vary with time, geographical position, radio frequency, etc.

\section{Channel estimation}

The radio channels in wireless communication system are usually multipath fading channels, in this situation, received signal is usually distorted by the channel characteristics. By placing spatially separated multiple antenna elements at both ends of the transmission link, MIMO technologies can improve the link reliability and provide a significant increase of the link capacity. In order to retrieve the transmitted bits, the channel effect must be estimated and compensated in the receiver. The location of channel estimation is figured out in Figure 1. In order to choose the channel estimation technique for the MIMOOFDM system under consideration, many different aspects of implementations, including the required performance, computational complexity and timevariation of the channel must be taken into account.

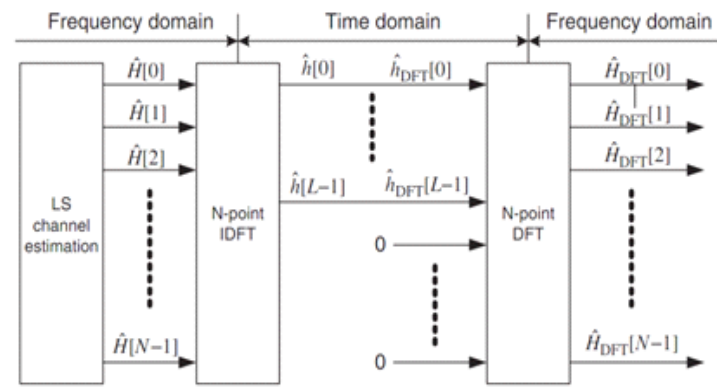

Fig. 2. DFT Based Channel Estimation 


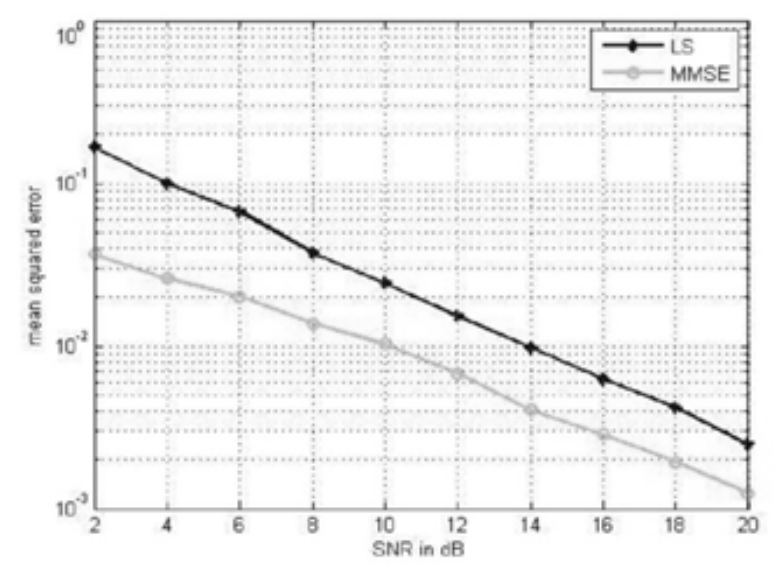

Fig. 3. Mean square error comparison

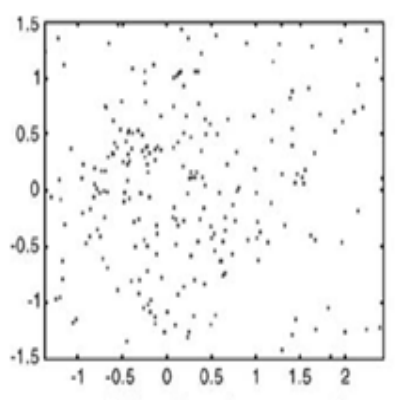

(a) Before channel compensation

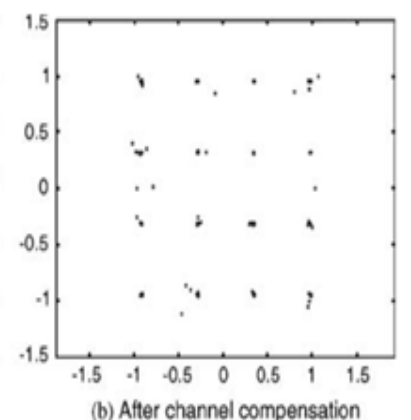

Fig. 5. Constellation diagram of received signal

The performance enhancement brought by MIMO systems mainly attributes to the space time techniques which aimed to remove the channel effects at both transmission and detection phase (Katalinic, et al 2006). Channel state information (CSI) generated by the channel estimator, is either fed back to the transmitter side to construct beamforming weight vector or sent into the detection block.

There are mainly four types of attributes to classify the channel estimator, which are pilot arrangement, estimation theory, processing domain and estimation iteration. According to pilot symbol arrangement the channel estimator are categorized into Block type, Comb type and Lattic type. Similarly in estimation theory, least square (LS) estimation and minimum mean square estimation (MMSE) are categorized (YushiShen \& Ed Martinez 2006).

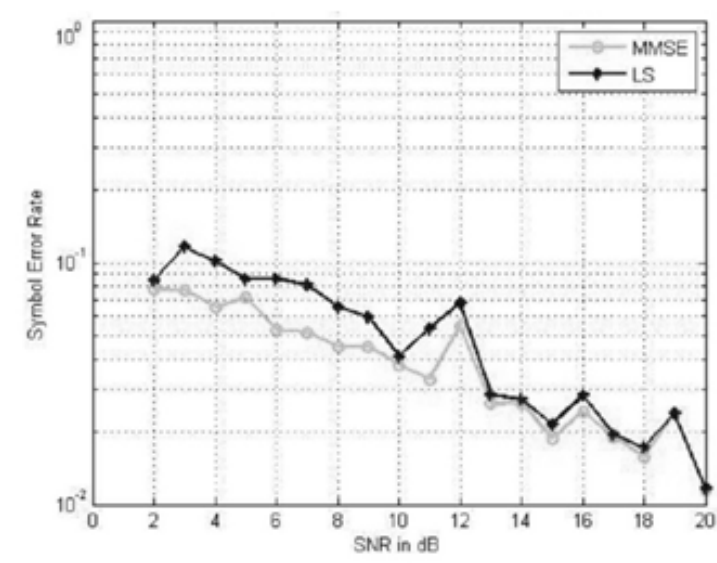

Fig. 4. Symbol error rate comparisons
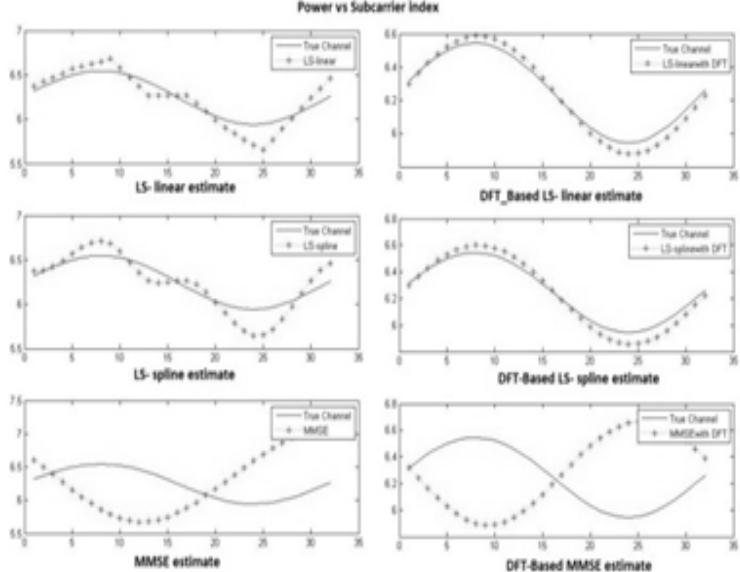

Fig. 6. Comparison with and without DFT-Based channel estimation

In general, the channel can be estimated by using a preamble or pilot symbols known to both transmitter and receiver, which employ various interpolation techniques to estimate the channel response of the subcarriers between pilot tones. In general, data signal as well as training signal, or both, can be used for channel estimation. Pilot structure is a signal usually in single frequency, transmitted over a communications channel for supervisory, control, equalization, continuity, synchronization, or reference purposes. Depending on the arrangement of pilots, three different types of pilot structures are considered: block type, comb type, and lattice type.

In block type pilot arrangement, OFDM symbols with pilots at all subcarriers are transmitted periodically for channel estimation and suitable for frequencyselective channels. Using these pilots, a time-domain 
interpolation is performed to estimate the channel along the time axis. Block-type pilot arrangement is suitable for frequency selective channels. In Combtype pilot arrangement, every OFDM symbol has pilot tones at the periodically-located subcarriers, which are used for a frequency domain interpolation to estimate the channel along the frequency axis. Comb type pilot arrangement is suitable for fast-fading channels. In Lattice-type pilot arrangement, pilot tones are inserted along both the time and frequency axes with given periods. The pilot tones scattered in both time and frequency axes facilitate time/frequencydomain interpolations for channel estimation. In order to keep track of the time-varying and frequencyselective channel characteristics, the pilot symbol arrangement must satisfy both Block type and Comb type inequality.

Training symbols based channel estimation usually providing a good performance. Overhead of training symbols like preamble symbol or pilot symbol reduce the transmission efficiencies. The least-square (LS) and minimum-mean-square-error (MMSE) techniques are widely used for channel estimation when training symbols are available. The least-square channel estimation method estimate the channel based on the least value of errors square without using any knowledge of the statistics of the channels, the LS estimators are calculated with very low complexity, but they suffer from a high mean-square error. MMSE channel estimation method estimate in terms of weight matrix. It employs the second-order statistics of the channel conditions to minimize the mean-square error. Error value is calculated from channel vector in such a way that the MSE is minimized.

\section{Least square channel estimation}

The principle of least squares is that the overall solution minimizes the sum of the squares of the errors made in solving every single equation. Compared to MMSE estimator LS estimator has lower computational complexity. It requires pilot symbols and matrix inversion. A training sequence is transmitted by each transmit antenna at the beginning of each data burst. The notation for transmitted signal is $\mathrm{x}$, received signal is $y$, the column in $F$ are orthogonal. The LS estimator for the cyclic impulse response $\mathrm{g}$ minimizes $\left(\mathrm{yxF}_{\mathrm{g}}\right)$ $\left(\mathrm{y} \text { “ } \mathrm{xF}_{\mathrm{g}}\right)^{\mathrm{H}}$ and generates the channel attenuation as below

$$
\begin{aligned}
& \mathrm{h}_{\mathrm{LS}}=\mathrm{FQ}_{\mathrm{LS}} \mathrm{F}^{\mathrm{H}} \mathrm{X}^{\mathrm{H}} \mathrm{y} \ldots \ldots \ldots \ldots \ldots . . .(\mathrm{i}) \\
& \text { where } \mathrm{Q}_{\mathrm{LS}}=\left(\mathrm{F}^{\mathrm{H}} \mathrm{X}_{\mathrm{H}}^{\mathrm{H}} \mathrm{Fx}\right)^{-1} \text { and } \\
& \left(\mathrm{y} \text { " } \mathrm{xF}_{\mathrm{g}}\right)^{\mathrm{H}} \text { are the conjugate transpose operations }
\end{aligned}
$$

Matrix operation hermitian and inverse are commonly used in this method. The least square channel impulse response or channel attenuation is written as $\mathrm{h}_{\mathrm{LS}}=\mathrm{x}^{-1} \mathrm{y}$

Hence, LS estimates are calculated without using any knowledge of statistics of the channel but they suffer from a high mean square error.

\section{Minimum mean square error channel estimation}

The MMSE estimator major rule is to efficiently estimate the channel to minimize the MSE or symbol error rate (SER) of the channel by employing secondorder statistics of the channel conditions. The constraints for MMSE channel estimation are autocorrelation matrix $\left(\mathrm{R}_{\mathrm{gg}}\right)$, auto-covariance matrix $\left(\mathrm{R}_{\mathrm{gy}}\right)$, noise variance $\left(\delta_{\mathrm{n}}\right)^{2}$. The notation for common signal are as follows, $x$ is the transmitted signal, $y$ is received signal, $g_{\text {MMSE }}$ is the channel energy(impulse response of channel), $h_{\text {MMSE }}$ is the channel attenuation (transfer function) for MMSE estimator and F is the DFT matrix.

The channel estimation by using MMSE estimator can be derived as follows:

$\mathrm{g}_{\text {MMSE }}=\mathrm{R}_{\mathrm{gy}} \mathrm{R}_{\mathrm{yy}}^{-1} \mathrm{y} \ldots \ldots \ldots \ldots \ldots \ldots$ (ii)
Where $\mathrm{R}_{\mathrm{gy}}=\mathrm{E}\left\{\mathrm{gy}^{\mathrm{H}}\right\}=\mathrm{R}_{\mathrm{gg}} \mathrm{F}^{\mathrm{H}} \mathrm{X}^{\mathrm{H}}$ and
$\mathrm{R}_{\mathrm{yy}}=\mathrm{E}\left\{\mathrm{yy}^{\mathrm{H}}\right\}=\mathrm{xFR}_{\mathrm{gg}} \mathrm{F}^{\mathrm{H}} \mathrm{X}^{\mathrm{H}}+\left(\mathrm{a}_{\mathrm{n}}\right)^{2} \mathrm{I}_{\mathrm{n}}$

Here the columns in $\mathrm{F}$ are orthogonal and In is the identity matrix of nth order. The channel impulse response is as follows:

$\mathrm{h}_{\text {MMSE }}=\mathrm{F}_{\mathrm{M}} \mathrm{g}_{\mathrm{MMSE}}=\mathrm{FQ}_{\mathrm{MMSE}} \mathrm{F}^{\mathrm{H}} \mathrm{x}^{\mathrm{H}} \mathrm{y} \ldots \ldots \ldots \ldots \ldots$.......ii)

where $\mathrm{Q}_{\mathrm{MMSE}}=\mathrm{R}_{\mathrm{gg}}\left[\left(\mathrm{F}^{\mathrm{H}} \mathrm{X}^{\mathrm{H}} \mathrm{XF}\right)\left(\delta_{\mathrm{n}}\right)^{2}+\mathrm{R}_{\mathrm{gg}}\right]^{-1}\left(\mathrm{~F}^{\mathrm{H}} \mathrm{X}^{\mathrm{H}} \mathrm{Fx}\right)-1$

Both estimators have their own drawbacks. However the LS estimator computational complexity is low, it has high mean-square error contrast to the MMSE estimator. Performance of estimator is tradeoff between the computational complexity and mean error. It is better to choose particular channel estimator based on requirement threshold and resource allocation.

\section{DFT-based channel estimation}

The DFT-based channel estimation technique is guided by the statement "the performance of LS or MMSE channel can be improved by eliminating the 
effect of noise outside the maximum channel delay”. Let $\mathrm{H}[\mathrm{K}]$ represent the estimate of channel gain at the $\mathrm{K}_{\text {th }}$ subcarrier, obtained by either LS or MMSE channel estimation method. Taking the IDFT of the channel estimate

$$
\text { IDFT }\{\mathrm{H}[\mathrm{K}]\}=\mathrm{h}[\mathrm{n}]+\mathrm{z}[\mathrm{n}] \triangleq \quad[\mathrm{n}]
$$

$\mathrm{n}=0,1, \ldots . . . \mathrm{N}-1$

where $\mathrm{z}[\mathrm{n}]$ the noise component in the time domain. Ignoring the coefficients $\mathrm{h}[\mathrm{n}]$ that contains the noise only and defining the coefficients for the maximum channel delay $L$ as $h_{D F T}[n]=\left\{\begin{array}{lr}h\lfloor n\rfloor+z\lfloor n\rfloor, n=0.1 .2 \ldots \ldots L-1 \\ 0, & \text { otherwise }\end{array}\right.$

and transform the remaining $L$ elements back to the frequency domain as follows

$\mathrm{H}_{\mathrm{DFT}}[\mathrm{K}]=\operatorname{DFT}\left\{\mathrm{h}_{\mathrm{DFT}}(\mathrm{n})\right\}$

The block diagram of DFT-based channel estimation followed by LS channel estimation theory is shown in Figure 2. The prerequisite condition for DFT-based channel estimation is that the maximum channel delay $L$ must be known in advance.

There are more channel estimation techniques with advance methods and logic. Decision Directed Channel Estimation technique uses the preamble or pilot signal only during beginning of channel estimation after that the coefficients of channel can be updated with decision directed feedback loop to track the possibility of time varying channel.

\section{Results and Discussion}

The simulation consists of two important parameter, called mean square error (MSE) and symbol error rate (SER), comparisons between LS and MMSE estimator with two main programs. The MSE of an estimator measures thedifference between the true value of the quantity being estimated and the theoretical values of an estimator. MSE evaluates the average of the square of the error. MSE is defined as

\footnotetext{
Mean square error

$=$ mean $\left[\left\{\text { abs }(\mathrm{H}) \check{S}_{\text {abs }}\left(\mathrm{h}_{\text {estimator }}\right)\right\}^{2}\right] \quad \ldots$ (vii)

Where, $\mathrm{H}$ is theoretical transfer function and $\mathrm{h}_{\text {estimator }}$ is the calculated transfer function for each estimator.
}

Number of symbol changes made to the transmission medium per second using a digitally modulated signal is called SER. Symbol error rate for 16-QAM system is

$P_{S E R-Q A M}=3 / 2 \operatorname{erfc}\left(\sqrt{\frac{E_{s}}{10 N_{0}}}\right)$.

Where, erfc denoted complementary error function, $\mathrm{E}_{\mathrm{s}}$ denotes signal energy and $\mathrm{N}_{0}$ denotes bit rate

The LS estimation, Frequency response from LS estimator is calculated by using input signal and theoretical value of signal. The impulse response of the LS estimator is calculated from MATLAB and used for calculation of mean square error.

In MMSE estimation, Auto-correlation function of channel energy, Auto-correlation function of output signal and Cross-correlation of the channel energy and output signal is calculated by using the MATLAB simulator. By using these values, impulse response function and the transfer function of estimator are calculated and finally using these function mean square error and symbol error rate is calculated.

The attribute used for simulation are FFT size 64, Number of sub-carrier 64, pilot ratio1:10, guard length 10 , guard type is cyclic prefix, bandwidth is $500 \mathrm{KHz}$ and modulation scheme is 16-Quadrature Amplitude Modulation (QAM).

In this simulation, the channel estimation based on time domain channel statistics is described. Using a general model for a slowly fading AWGN channel, we present the MMSE and LS estimators. The MSE and SER for a BPSK system is demonstrated by means of simulation. Depending upon estimator complexity in MSE performance, up to $4 \mathrm{~dB}$ in SNR can be gained in MMSE estimator over the LS estimator in $1 \%$ probability of error. In SER simulation both estimator have nearly same characteristics.

Figure 5 illustrates the effect of channel estimation by plotting the received signal constellation before and after channel compensation for the OFDM system with 16-QAM. In the same manner Figure 6 illustrates the channel estimates obtained by using 
the various types of channel estimation methods with and without DFT technique.

Based on the simulation of DFT-Based channel estimator on LS- linear, LS-spline and MMSE estimation all three show the better performance than DFT not using channel estimator. Channels are generated by using Gaussian random number function "randn." It is also seen that MMSE estimator has better performance than LS estimator at the trade of requirement of additional computing and channel characteristics information.

From the comparison of channel estimation technique between LS and MMSE, MSE performance of MMSE estimator has $4 \mathrm{~dB}$ gained in SNR at 1\% probability of error. From the simulation of DFT-Base channel estimation technique the statement "the performance of LS or MMSE channel can be improved by eliminating the effect of noise outside the maximum channel delay" is verified and all three channel estimator LS- linear, LS-spline and MMSE estimation show the better performance after DFT-Based channel estimation.

\section{Acknowledgements}

This research was conducted under the supervision and guidance of Department of Electronics and Computer Engineering of Institute of Engineering, Pulchowk Campus. We thank to our thesis supervisor Prof. Shashidhar Ram Joshi for his continuous support during the study.

\section{References}

Aguiar, Ana and James Gross.2003.Wireless Channel Models. PhD dissertation.Technical University Berlin, Germany.

Katalinic, A., R. Nagy and R. Zentner. 2006.Benefits of MIMO systems in practice: Increased capacity, reliability and spectrum efficiency. In: 48th International Symposium ELMAR-2006 focused on Multimedia Signal Processing and Communications. University of Zagreb, Zadar, Croatia, pp. 7-9.

Kowalski, Rony. 2007. The Benefits High Dynamic Adaptive Modulation for High Capacity of Wireless Backhaul Solutions.White paper, Ceragon Networks Centre, Tel Aviv, Israel, pp. 3-5.

Yushi, Shen and Ed. Martinez. 2006. Channel Estimation in OFDM Systems.Freescale Semiconductor Application Note.Rev 0, 1/2006, pp 9. 